\title{
Recommendations for the Diagnosis and Treatment of Endometrial Cancer, Update 2013
}

\author{
Empfehlungen zur Diagnostik und Therapie des Endometriumkarzinoms, \\ Update 2013
}

Authors

Affiliations
G. Emons ${ }^{1}$, P. Mallmann ${ }^{2}$, acting for the Uterus Commission of AGO

${ }^{1}$ Universitäts-Frauenklinik, Gynäkologisches Krebszentrum, Göttingen

${ }^{2}$ Universitäts-Frauenklinik, Gynäkologisches Krebszentrum, Köln
Deutschsprachige Zusatzinformationen online abrufbar unter: www.thieme-connect.de/ ejournals/toc/gebfra
First Publication

First published in Frauenarzt 2013; 54: 660-664
Bibliography

DOI http://dx.doi.org/

$10.1055 / \mathrm{s}-0034-1368268$

Geburtsh Frauenheilk 2014; 74 : 244-247 @ Georg Thieme Verlag KG Stuttgart · New York . ISSN 0016-5751

\section{Correspondence}

Prof. Dr. med. Günter Emons,

Director of the University

Gynecological Hospital

Gynäkologisches Krebszentrum

Robert-Koch-Straße 40

37075 Göttingen

emons@med.uni-goettingen.de

\section{Introduction}

$\checkmark$

The S2k guideline "Diagnosis and Treatment of Endometrial Cancer" was published on June 1, 2008; some of the recommendations in the guideline are still up-to-date; however, others are out-of-date and no longer apply. The update will be carried out in accordance with the requirements of the German National Cancer Plan and will lift the guideline into the S3 category. We expect that the funding required for the update will be available from the 2 nd half of this year. Even if work starts promptly, the S3 guideline will only be available from the beginning of 2016 at the earliest. As an interim solution for the period for which no guideline is available, the Uterus Commission (O Table 2) has issued recommendations for the diagnosis and treatment in April 2011, which were since updated in April 2013. The FIGO/TNM staging system (O Table 1 ) was used.

\section{Early Detection and Screening}

$\nabla$

1. Screening should not be carried out in asymptomatic women who have no risk factors.

2. There is no evidence that screening of high-risk populations reduces mortality.

\section{Diagnosis}

1. The diagnosis of endometrial cancer and its precursors must be confirmed by histological sampling.

2. Every post-menopausal bleeding must be investigated irrespective of endometrial thickness on ultrasound.

3. Atypical bleeding in perimenopausal women should be investigated histologically.
4. Only bleeding requires histological investigation in women receiving tamoxifen.

5. There are currently no imaging methods which could replace surgical staging in endometrial cancer. Magnetic resonance imaging should be done to plan radiotherapy for women whose cancer is inoperable due to comorbidities.

6. Histological classification of endometrial cancers and their precursors follows the WHO criteria.

7. Any report of histopathological findings for endometrial cancer must include: tumor type, grading, depth of invasion into the myometrium, invasion of stromal connective tissue of the cervix, lymph node involvement if applicable, $R$ classification, lymphatic and vascular metastasis, and nerve sheath invasion.

\section{Patient Information}

$\nabla$

1. The information provided to patients (print or internet media) must be compiled in accordance with the quality requirements of the Guideline on Information Provided to Women to support every patient in her autonomous decision for or against medical treatment by communicating the risks involved in a comprehensible form (information should list incidence rather than relative percentage).

2. Information provided to patients should be both comprehensive and truthful; the information should be presented clearly and in accordance with the principles of patient-centered communication listed here:

- Show empathy and demonstrate active listening

- Address difficult topics directly and sensitively

- If possible, avoid specialist medical terminology; explain technical terms where necessary 
Table 1 The new FIGO/TNM classification for endometrial cancer.

\begin{tabular}{|c|c|c|}
\hline TNM categories & FIGO stages & Definitions \\
\hline TX & & Primary tumor cannot be assessed \\
\hline T0 & & No evidence of primary tumor \\
\hline T1 & $1^{1}$ & Tumor confined to corpus uteri \\
\hline T1a & $\mathrm{IA}^{1}$ & Tumor limited to endometrium or invades less than one half of the myometrium \\
\hline T1b & IB & Tumor invades one half or more of the myometrium \\
\hline $\mathrm{T} 2$ & II & Tumor invades stromal connective tissue of the cervix but does not extend beyond uterus \\
\hline T3 and/or N1 & III & Local and/or regional extension or metastasis as described below: \\
\hline T3a & IIIA & Tumor involves serosa or adnexa (direct extension or metastasis) \\
\hline $\mathrm{T} 3 \mathrm{~b}$ & IIIB & Vaginal involvement or parametrial involvement (direct extension or metastasis) \\
\hline \multirow[t]{3}{*}{ N1 } & IIIC & Metastasis to pelvic and/or para-aortic lymph nodes ${ }^{2}$ \\
\hline & IIIC1 & Metastasis to pelvic lymph nodes \\
\hline & IIIC2 & Metastasis to para-aortic lymph nodes \\
\hline T4 & IVA & Tumor invades bladder mucosa and/or bowel mucosa ${ }^{3}$ \\
\hline M1 & IVB & $\begin{array}{l}\text { Distant metastasis (excludes metastasis to the vagina, pelvic serosa or adnexa; includes metastasis } \\
\text { to inguinal and intra-abdominal lymph nodes other than para-aortic and/or pelvic lymph nodes) }\end{array}$ \\
\hline
\end{tabular}

1 Endocervical glandular involvement should only be considered as stage I.

2 Positive cytology must be diagnosed separately and recorded without changing the stage.

${ }^{3}$ Bullous edema is not sufficient to classify a tumor as T4. Invasion of bladder mucosa or bowel mucosa must be confirmed with biopsy.

- Use strategies to improve patient's understanding (repetition, summary of most important information, use of graphs, etc.)

$\checkmark$ Encourage patients to ask questions

- Permit and encourage patients to express their feelings

- Offer additional help (for example, support groups, psychooncology, psychosocial cancer counselling)

\section{Treatment}

\section{$\nabla$}

Treatment of precursors of endometrial cancer

1. Women with endometrial hyperplasia without atypia can be treated conservatively.

2. Atypical endometrial hyperplasias have a higher risk of evolving into cancerous lesions and a higher rate of synchronous cancers.

3. Pre-menopausal and post-menopausal women with atypical endometrial hyperplasia should undergo hysterectomy.

4. Women with atypical endometrial hyperplasia should only be treated conservatively if they wish to have children and are prepared to undergo regular close monitoring and repeat biopsies.

\section{Treatment of early endometrial cancer}

1. Conservative treatment with high-dose gestagens can be considered for women with well-differentiated, progesterone receptor-positive, endometrioid endometrial cancer, FIGO stage IA without invasion of the myometrium who strongly wish to have children.

2. Conservative treatment should only be considered if the patient strongly wishes to have children and is prepared to undergo regular close monitoring and repeat biopsies.

3. Because of the high risk of recurrence, women who have completed their family should have a hysterectomy.

\section{Surgical treatment for endometrial cancer}

1. Surgical treatment of endometrial cancer should consist of cytological evaluation of the abdominal cavity, hysterectomy and bilateral extirpation of the adnexa.
2. If lymph nodes are macroscopically unremarkable intraoperatively, no lymphadenectomy should be done in patients with endometrioid cancer stage pT1a, G1 or G2.

3. Pelvic and para-aortic lymphadenectomy up to the renal pedicle should be carried out when treating tumors associated with a higher risk of invasion of retroperitoneal lymph nodes (stage $\geq$ pT1b, all G3 or serous or clear cell cancers).

4. Laparoscopic procedures have a lower short-term postoperative morbidity compared to open surgery. The long-term morbidity is the same for both approaches.

5. In low risk cases, the oncological outcome of laparoscopic treatment is comparable with that of open surgery. There is not enough data available to compare oncological outcomes in high-risk cases.

6. Multiple peritoneal biopsies and omentectomy should additionally be done if serous or clear cell carcinoma is present. This should also be done for endometrioid cancers with macroscopic evidence of stage cT3a at surgery.

7. In patients with advanced disease, complete resection of all tumor manifestations should be aimed for, as this improves the efficacy of adjuvant systemic therapy and radiation therapy.

8. Radical hysterectomy should not be carried out to treat stage II endometrial cancer.

\section{Radiation therapy}

1. Primary radiation treatment of the endometrial cancer is indicated if the cancer is considered inoperable due to the patient's comorbidities.

2. Adjuvant radiation therapy is not indicated to treat patients with a low risk of local recurrence (endometrioid histology, stage Ia, G1-2).

3. Patients with a medium risk of local recurrence (endometrioid histology and stage Ia, G3, as well as stage Ib G1-G2) should receive adjuvant brachytherapy.

4. Patients with a high risk of local recurrence (stage Ib, G3 and stage II) should receive adjuvant brachytherapy or teletherapy.

5. Patients with advanced stage III disease should receive adjuvant teletherapy or brachytherapy.

6. Adjuvant or palliative radiation therapy (teletherapy) may be indicated for stage IV disease. 
7. Randomized studies found a significant reduction of locoregional recurrence in patients with stage I and II disease after adjuvant radiation therapy, but this had no significant impact on overall survival. The available data for advanced disease is insufficient to permit any recommendations to be made.

\section{Systemic adjuvant therapy}

1. Patients should not receive adjuvant endocrine therapy with gestagens.

2. Patients with stage IB G3, II and III disease and all patients with serous or clear cell endometrial cancer should receive chemotherapy sequential to radiation therapy (brachytherapy and/or teletherapy). The most studied chemotherapy regimen consists of a combination of platinum-based chemotherapy plus paclitaxel.

3. Patients with stage IVA can undergo chemotherapy and/or sequential radiation therapy after surgery. The most studied chemotherapy regimen consists of a combination of platinumbased chemotherapy plus paclitaxel. However, the optimal combination and sequence have not yet been defined for this combination.

\section{Palliative systemic therapy}

1. Endocrine therapy with gestagens should be offered to patients with estrogen and/or progesterone receptor-positive cancer who have inoperable recurrence or metastasis not amenable to radiation therapy if metastases are not acutely lifethreatening.

2. Palliative chemotherapy can be offered to patients who present with disease progression despite endocrine therapy and to patients with receptor-negative tumors or acutely life-threatening tumors. Effective substances include anthracyclines, platinum derivatives and taxanes.

3. Combination chemotherapy regimens have better response rates and progression-free survival rates compared to monotherapies, but the benefits in terms of overall survival are only marginal. The combination of carboplatin/paclitaxel should be used if the patient can sufficiently tolerate the regimen as the efficacy of this regimen is the same as for the combination of adriamycin/cisplatin/paclitaxel but toxicity is lower.

\section{Recurrence and metastasis}

1. Surgery is the primary treatment for resectable locoregional and intra-abdominal recurrence if complete resection is possible.

2. Radiation therapy is the treatment of choice for inoperable locoregional recurrence.

3. Palliative systemic therapy can be offered to patients if surgery or radiation therapy of the local recurrence are not possible or are not oncologically useful.

4. If extra-abdominal distant metastasis is present, the choice of therapy - surgery, radiation therapy or systemic therapy must be decided individually, on a case-by-case basis.

\section{Supportive therapy}

1. Guideline-based supportive therapy to prevent or minimize therapy- or tumor-induced symptoms is necessary.
Table 2 Uterus Commission of AGO of the DGGG (German Society for Gynecology and Obstetrics) and the DKG (German Cancer Society).

\begin{tabular}{l} 
Gynecological Oncology \\
- Prof. Dr. M. W. Beckmann, Erlangen \\
- Prof. Dr. D. Denschlag, Bad Homburg \\
- Prof. Dr. G. Emons, Göttingen \\
- Priv.-Doz. Dr. M. Fleisch, Düsseldorf \\
- Prof. Dr. P. Hillemanns, Hannover \\
- Dr. I. Juhasz-Böss, Homburg/Saar \\
- Prof. Dr. L. Kiesel, Münster \\
- Prof. Dr. R. Kimmig, Essen \\
- Priv.-Doz. Dr. C. Köhler, Berlin \\
- Prof. Dr. B. Lampe, Düsseldorf \\
- Prof. Dr. W. Lichtenegger, Berlin \\
- Prof. Dr. P. Mallmann, Cologne (Commission spokesman) \\
- Priv.-Doz. Dr. H. Pilch, Cologne \\
- Priv.-Doz. Dr. D. Rein, Cologne (Deputy Commission spokesman) \\
- Prof. Dr. I. Runnebaum, Jena \\
- Priv.-Doz. Dr. E. Steiner, Rüsselsheim \\
- Dr. H. Strauss, Halle/Saale \\
- Prof. Dr. C. Tempfer, Herne \\
- Prof. Dr. U. Ulrich, Berlin \\
Radiation Oncology \\
- Prof. Dr. S. Marnitz, Berlin \\
- Prof. Dr. V. Strnad, Erlangen \\
- Prof. Dr. D. Vordermark, Halle/Saale (ARO representative) \\
Pathology \\
- Prof. Dr. L. C. Horn, Leipzig \\
- Prof. Dr. D. Schmidt, Mannheim \\
\hline
\end{tabular}

\section{Psycho-oncology}

$\nabla$

1. Offering psycho-oncological care to patients with endometrial cancer is an integral part of oncological diagnosis, treatment, rehabilitation and follow-up care and requires interdisciplinary cooperation.

2. Patients should be informed early on about the different types of in-patient and outpatient psycho-oncological support available and should receive professional psycho-oncological care if required.

3. The patient's quality of life must be assessed regularly during treatment, rehabilitation and follow-up, as this allows physicians to assess whether psycho-oncological action is required.

\section{Rehabilitation}

1. All patients must be informed and advised by their attending physician about the statutory options for rehabilitation/convalescence following in-patient treatment and the available outpatient rehabilitation opportunities.

\section{Follow-up}

$\nabla$

1. As early detection of local recurrence is necessary for curative resection, patients should be followed up every 3 months in the first 2-3 years after primary therapy by speculum examination, vaginal and rectal examination, and ultrasound, where required.

2. Additional imaging for diagnostic purposes is only necessary for symptomatic patients. 
3. Follow-up consultations should address the topics listed below:

- transient and long-term impact of disease and therapy

- psycho-oncological/psychotherapeutic treatment options

$\checkmark$ sexuality and relationships

quality of life

These recommendations are also available on the AGO website (www.ago-online.de).

\section{Conflict of Interest}

There is no conflict of interest. 